\title{
Amnesia in Frontotemporal Dementia with Amyotrophic Lateral Sclerosis, Masquerading Alzheimer's Disease
}
A. Yamanami-Irioka ${ }^{a, c}$
T. Uchihara ${ }^{a}$
T. Endo ${ }^{b}$
T. Irioka ${ }^{c}$
M. Watanabe ${ }^{c}$
M. Kitagawab
H. Mizusawa ${ }^{\mathrm{C}}$

a Laboratory of Structural Neuropathology, Tokyo Metropolitan Institute of Medical Science, and Departments of ${ }^{\mathrm{b}}$ Pathology and ${ }^{\mathrm{C}}$ Neurology, Tokyo Medical and Dental University, Tokyo, Japan

\section{Key Words}

Amyotrophic lateral sclerosis $\cdot$ Dementia $\cdot$ Alzheimer's disease $\cdot$ Creatine kinase

\section{Abstract}

A 68-year-old man with a clinical diagnosis of Alzheimer's disease (AD) later developed amyotrophic lateral sclerosis (ALS), which was confirmed at autopsy at age 72 years. Because neuronal loss and AD-type pathologies (Braak stage II for neurofibrillary tangles) were scant, TDP-43-positive intracytoplasmic inclusions in hippocampal dentate granular cells and in neurons in the subiculum and amygdala, even though small in amount, may represent the earliest lesions of ALS-related dementia and could be the cause of dementia in this patient. Although the persistent elevation of creatine kinase from the onset could be a pointer to the presence of motor involvement, more accurate characterization of dementia, which may differentiate ALS-related dementia and AD, is necessary.

\section{Introduction}

The association of dementia with amyotrophic lateral sclerosis (ALS) is attracting increasing attention, especially after the identification of TDP-43 [1, 2], which may provide a molecular link between dementia and motor involvement. However, clinical features of dementia in ALS patients are too heterogeneous to be differentiated from other disorders accompanied with dementia. Some of these patients show memory impairments indistinguishable from AD-type dementia [3]. Ubiquitin/TDP-43-positive, tau-negative intraneuronal inclusions in the hippocampal dentate granular cells and 
neurons of the transentorhinal cortex are candidates to explain memory impairments in ALS [3]. Here, we report an autopsy-confirmed ALS patient with an onset of memory impairments, diagnosed as AD-type dementia 3 years prior to the onset of ALS. Because AD-related lesions were scant, extramotor lesions, although limited to some TDP-43positive intraneuronal inclusions in the dentate gyrus, subiculum and amygdala, may be more plausible candidate lesions to explain the cognitive impairments in this ALS patient.

\section{Case Report}

A 68-year-old man was admitted to the endocrinology department of our hospital for the treatment of isolated ACTH deficiency. He had undergone coronary bypass graft for ischemic heart disease and pacemaker implantation because of recurrent attacks of syncope. He presented with severe delirium on the day of admission, which subsided after restoration of serum cortisone (1.11$4.76 \mu \mathrm{g} / \mathrm{dl}$; reference range 6.4-21) and sodium (118-130 mEq/l; reference range 138-146). Even after recovery from this episode of delirium, a neurologist and a psychiatrist found his memory impairment and apathy were persistent. The patient's Hasegawa Dementia Scale score (revised Japanese version; HDS-R) [4] was 21/30 at the age of 68 years. Enlargement of inferior horns of lateral ventricles with medial temporal lobe atrophy shown on brain CT (fig. 1a) was compatible with the clinical diagnosis of possible AD (NINCDS-ADRDA criteria) [5]. Brain SPECT showed hypoperfusion of the bilateral parietal lobe, but in the frontal, temporal, and posterior cingulate lesions, AD-characteristic changes were not evident (fig. 1b).

Three years later, at the age of 72 years, the patient complained of muscle weakness and dysphagia, even though ACTH deficiency was stable and well controlled. On admission to our neurology department, upper and lower motor neuron involvement in the cranial region and all limbs was noted. Needle electromyography demonstrated spontaneous activities and a poor interference pattern with long-duration, high-amplitude potentials in the muscles examined. The clinical diagnosis of definite ALS was made. In addition to memory disturbance (HDS-R 20/30), delayed recall and category fluency were impaired. Moreover, indifference and apathy became more evident. Although abnormal behaviors were not apparent, he refused taking liquid food and tracheal suction, in spite of severe dysphagia. On brain CT repeated at the age of 72 years (fig. 1c), ischemic changes were not evident, and progression of atrophy was not remarkable in comparison to the brain CT performed at the age of 68 years. Serum creatine kinase was persistently elevated up to $575 \mathrm{IU} / \mathrm{l}$ (200-330; reference range 35-175) during the entire clinical course of 4 years.

The patient suffered from acute respiratory failure and died of pneumonia at the age of 72 years. His clinical course of dementia lasted 4 years, with additional motor symptoms during the last year. A general autopsy disclosed generalized atrophy of muscles, including respiratory muscles and bronchopneumonia, as an immediate cause of death.

The brain weighed 1,270 g after fixation. Macroscopically, thin diaphragms and anterior spinal roots were noted. Transverse section of the cervical cord stained with the Klüver-Barrera method disclosed mild degeneration of bilateral pyramidal tracts (fig. 1d). Microscopic findings included mild spongiosis in layer II of the precentral cortex and fallout of motor neurons in the anterior horn and cranial nuclei. Bunina bodies, skein-like inclusions positive for ubiquitin and cytoplasmic inclusions positive for phosphorylated TDP-43 were also found in these lower motor nuclei (fig. 1e-h). In contrast with these definite pathological changes of ALS, neurofibrillary tangle (NFT) stage according to Braak was II and A $\beta$-positive deposits were rare, the quantity hardly explaining the clinically overt dementia. $\alpha$-Synuclein deposits and argyrophilic grains were absent throughout the brain. Neuronal loss was restricted to the precentral cortex, where no NFTs were found. Although the nucleus basalis of Meynert, basal ganglia, thalamus, substantia nigra, white matter and cortices in the frontal, temporal and parietal lobes and amygdala were well preserved, hippocampal dentate granular cells contained a few neuronal cytoplasmic inclusions positive for phosphorylated TDP-43 but negative for ubiquitin (fig. 1i). Similar inclusions were found in the subiculum and amygdala. 


\section{Discussion}

This patient had a 3-year clinical history of AD-type dementia before being diagnosed as having ALS, which was ultimately confirmed at autopsy based on cytopathological changes characteristic of ALS, such as Bunina bodies, TDP-43-positive inclusions and skein-like inclusions. Initial memory impairment with bilateral hippocampal atrophy naturally led to the clinical diagnosis of AD [5], as interpreted by two independent specialists (neurologist and psychiatrist) at a university hospital. Neuropathological examination, however, did not support this diagnosis because NFTs were scant (Braak stage II) and A $\beta$-positive deposits were rare. Lewy bodies, argyrophilic grains and Pick bodies were absent. Although these age-related, quantitative changes were not abundant enough to explain overt clinical dementia, extramotor lesions possibly linked to cognitive impairment in ALS were found in form of TDP-43-positive intracytoplasmic inclusions in the hippocampal dentate granular cells and neurons in the subiculum and amygdala; in contrast, the neuritic profile was scant, compatible with type 2 according to the Sampathu-Neumann classification [2]. In addition to these qualitative cellular markers for ALS and ALS-related dementia, restricted spongiosis was found in the premotor cortex, where NFTs and $A \beta$ deposits were absent, again suggestive of ALS.

Cognitive deficits associated with ALS can be highly variable [6], and clinical differentiation from other causes of dementia is quite a challenge. Although it is generally considered as frontotemporal dementia of non-AD type [7], more specific clinical clues have been proposed [8] but have not always been found to be useful to characterize ALS-related dementia. Moreover, memory impairment as an initial manifestation of dementia in ALS patients has been reported [9], and the possible association of AD pathology with ALS may further complicate the interpretation [10]. Since the involvement of the so-called hippocampal perforant pathway in ALS could be severe enough to cause memory impairments in some ALS patients even in the absence of AD pathology [3], memory impairment as an initial manifestation of dementia does not necessarily favor the diagnosis of $\mathrm{AD}$ as in our patient.

In retrospect, cognitive impairment of this patient, initially compatible with $\mathrm{AD}$, has evolved over the next 3 years, and he showed indifference and apathy even when his HDS-R score still remained 20. Although this progressive course suggests some degenerative process, it is surprising that AD lesions were scant and ALS-related pathology in relevant areas was minimal. Currently, diagnostic criteria for ALS-related dementia are not yet available, probably because of the heterogeneity in this disease's clinical manifestations and the diversity of its pathological features [11]. However, the presence of TDP-43-positive intracytoplasmic inclusions in the dentate gyrus indicates extramotor extension of ALS-related lesions. Because similar inclusions could be present even in ALS patient without overt dementia [12], it is not yet possible to decide whether the ALS-related inclusions in this patient may explain his cognitive deficits. Considering the absence of other specific cytopathologies, however, we are left with the possibility that the extramotor pathology of this patient represents the earliest lesions of dementia related to ALS. This interpretation paves the way for early therapeutic intervention in ALS-related dementia before neuronal depletion and specific cytopathologies are florid. It is of note that creatine kinase was elevated throughout the disease course, which may alert the presence of ALS but usually is not seen in AD 
patients. Indeed, a patient with non-AD-type dementia with elevated creatine kinase was found to have ALS-related dementia, even though motor deficits were clinically absent throughout the course [13]. This awareness might have prompted examination with needle electromyography at an earlier stage even before motor deficits were manifesting.

In summary, we report an autopsy-confirmed ALS patient. Because AD pathology was too scant to explain his initial amnesia masquerading AD, extramotor lesions of ALS, although limited, may represent the earliest stage of dementia related to ALS, which may allow early therapeutic intervention hopefully available in the near future.

\section{Acknowledgements}

The authors are grateful to Ms. Ayako Nakamura for her excellent preparation of histological sections. This study is supported by Grants-in-Aid for Scientific Research (22500325, 22240039 TU) from the Ministry of Education, Culture, Sports, Science and Technology, from Mitusi Life Science Welfare Foundation, and from Comprehensive Research on Disability Health and Welfare, Ministry of Health, Labour and Welfare. 

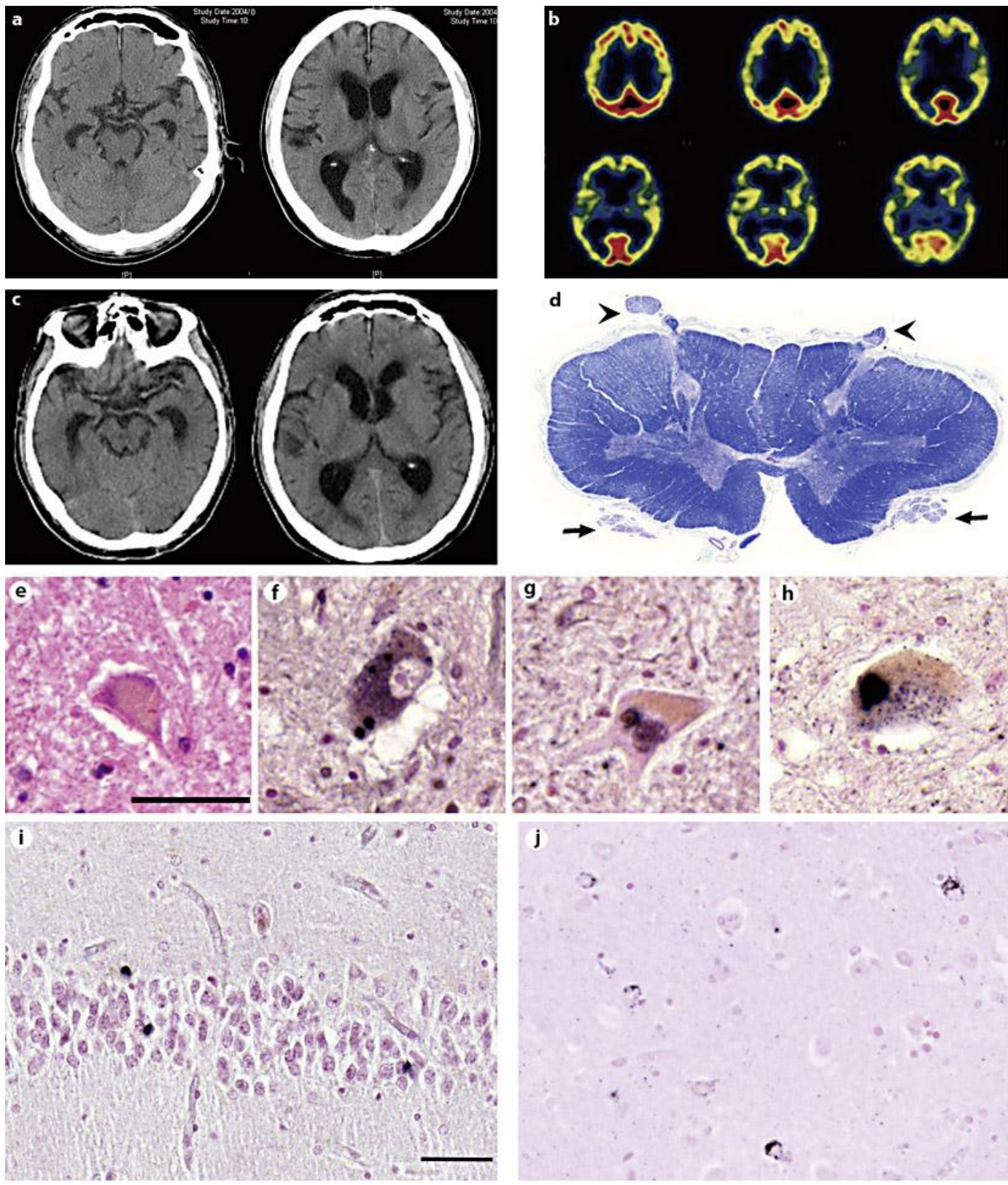

Fig. 1. a Brain CT at age 68. Enlargement of bilateral inferior horns of lateral ventricles with mild medial temporal lobe atrophy. b Brain SPECT at age 68. Hypoperfusion of the bilateral parietal lobe. c Brain CT at age 72. Progression of atrophy was not remarkable in comparison to a. $\mathbf{d}$ Cervical spine. Bilateral mild pyramidal tracts degeneration. The arrows and arrowheads point to the anterior and posterior roots, respectively. e-h ALS-specific cytoplasmic inclusions in lower motor neurons. Bar: 50 $\mu \mathrm{m}$. e, $\mathbf{f}$ Bunina bodies in a hypoglossal nucleus (e) and lumbar spinal motor neuron immunopositive for cystatin C (f). $\mathbf{g}$ Skein-like inclusion in a hypoglossal nucleus immunopositive for ubiquitin. h Cytoplasmic inclusion in a lumbar spinal motor neuron immunopositive for phosphorylated TDP-43. i, j Cytoplasmic inclusions immunopositive for TDP-43 in the hippocampal dentate gyrus (i) and amygdala (j). Bar: $50 \mu \mathrm{m}$. d Klüver-Barrera stain. e Hematoxylin and eosin stain. $\mathbf{f}$ Immunostain with anti-cystatin C. $\mathbf{g}$ Immunostain with anti-ubiquitin. $\mathbf{h}-\mathbf{j}$ Immunostain with anti-phosphorylated TDP-43. 


\section{References}

-1 Arai T, Hasegawa M, Akiyama H, Ikeda K, Nonaka T, Mori H, Mann D, Tsuchiya K, Yoshida M, Hashizume Y, Oda T: TDP-43 is a component of ubiquitin-positive tau-negative inclusions in frontotemporal lobar degeneration and amyotrophic lateral sclerosis. Biochem Biophys Res Commun 2006;351:602-611.

2 Neumann M, Sampathu DM, Kwong LK, Truax AC, Micsenyi MC, Chou TT, Bruce J, Schuck T, Grossman M, Clark CM, McCluskey LF, Miller BL, Masliah E, Mackenzie IR, Feldman H, Feiden W, Kretzschmar HA Trojanowski JQ, Lee VM: Ubiquitinated TDP-43 in frontotemporal lobar degeneration and amyotrophic lateral sclerosis. Science 2006;314:130-133.

-3 Takeda T, Uchihara T, Arai N, Mizutani T, Iwata M: Progression of hippocampal degeneration in amyotrophic lateral sclerosis with or without memory impairment: distinction from Alzheimer disease. Acta Neuropathol 2009;117:35-44.

-4 Kim KW, Lee DY, Jhoo JH, Youn JC, Suh YJ, Jun YH, Seo EH, Woo JI: Diagnostic accuracy of mini-mental status examination and revised hasegawa dementia scale for Alzheimer's disease. Dement Geriatr Cogn Disord 2005;19:324-330.

5 McKhann G, Drachman D, Folstein M, Katzman R, Price D, Stadlan E: Clinical diagnosis of Alzheimer's disease: report of the NINCDS-ADRDA Work Group under the auspices of Department of Health and Human Services Task Force on Alzheimer's Disease. Neurology 1984;34:939-944.

6 Phukan J, Pender NP, Hardiman 0: Cognitive impairment in amyotrophic lateral sclerosis. Lancet Neurol 2007;6:994-1003.

7 Mitsuyama Y, Inoue T: Clinical entity of frontotemporal dementia with motor neuron disease. Neuropathology 2009;29:649-654.

$>8$ Bozeat S, Gregory CA, Ralph MA, Hodges JR: Which neuropsychiatric and behavioural features distinguish frontal and temporal variants of frontotemporal dementia from Alzheimer's disease? J Neurol Neurosurg Psychiatry 2000;69:178-186.

-9 Mitsuyama Y, Takamiya S: Presenile dementia with motor neuron disease in Japan. A new entity? Arch Neurol 1979;36:592-593.

10 Rusina R, Sheardová K, Rektorová I, Ridzon P, Kulist'ák P, Matej R: Amyotrophic lateral sclerosis and Alzheimer's disease - clinical and neuropathological considerations in two cases. Eur J Neurol 2007;14:815-818.

11 Yoshida M: Amyotrophic lateral sclerosis with dementia: the clinicopathological spectrum. Neuropathology 2004;24:87-102.

12 Tsuchiya K, Takahashi M, Shiotsu H, Akiyama H, Haga C, Watabiki S, Taki K, Nakano I, Ikeda K: Sporadic amyotrophic lateral sclerosis with circumscribed temporal atrophy: a report of an autopsy case without dementia and with ubiquitinated intraneuronal inclusions. Neuropathology 2002;22:308-316.

13 Uchihara T, Sato T, Suzuki H, Ikeda K, Akiyama H, Takatori T: Bunina body in frontal lobe dementia without clinical manifestations of motor neuron disease. Acta Neuropathol 2001;101:281-284 